

\title{
Problèmes relatifs à l'étalonnage d'un spectromètre à grilles à haute résolution
}

\author{
J. Botineau
}

\section{To cite this version:}

J. Botineau. Problèmes relatifs à l'étalonnage d'un spectromètre à grilles à haute résolution. Revue de Physique Appliquée, 1970, 5 (6), pp.829-834. 10.1051/rphysap:0197000506082900 . jpa-00243461

\section{HAL Id: jpa-00243461 https://hal.science/jpa-00243461}

Submitted on 1 Jan 1970

HAL is a multi-disciplinary open access archive for the deposit and dissemination of scientific research documents, whether they are published or not. The documents may come from teaching and research institutions in France or abroad, or from public or private research centers.
L'archive ouverte pluridisciplinaire HAL, est destinée au dépôt et à la diffusion de documents scientifiques de niveau recherche, publiés ou non, émanant des établissements d'enseignement et de recherche français ou étrangers, des laboratoires publics ou privés. 


\title{
PROBLÈMES RELATIFS A L'ÉTALONNAGE D'UN SPECTROMẼTRE A GRILLES A HAUTE RÉSOLUTION (*)
}

\author{
par J. BOTINEAU \\ Office National d'Etudes et de Recherches Aérospatiales, \\ 92, Châtillon-sous-Bagneux, France
}

(Reçu le 3 juillet 1970)

\begin{abstract}
Résumé. - Les conditions à remplir par un système d'étalonnage en longueur d'onde sont passées en revue et le dispositif expérimental adopté sur le spectromètre à grilles ONERA est exposé. A titre d'exemple, quelques extraits du spectre de la bande $v_{4}$ du méthane sont commentés.

Abstract. - The conditions to be fulfilled by a wavelength calibration set are examined and the experimental device adopted for the ONERA dual-grille spectrometer is exposed. Several portions of the $v_{4}$ band methane of the spectrum are commented.
\end{abstract}

Introduction. - Le spectre des états d'énergie d'une molécule est déterminé par la symétrie et les constantes moléculaires, ainsi que par le mode d'excitation utilisé. Inversement, un spectre enregistré dans des conditions définies permet de déterminer la symétrie et une partie des constantes de la molécule. Pour cela il est important d'étalonner le spectre de façon aussi précise que possible, ce qui implique deux conditions :

- la résolution du spectromètre doit être suffisamment élevée pour séparer toutes les raies composantes. Cette condition est rarement réalisée, surtout dans les branches du type $Q$.

- Les points de référence de l'étalonnage doivent être déterminés avec une précision en rapport avec la résolution de l'appareil.

Dans ce qui suit, seul le cas des spectres infrarouges en absorption est étudié.

\section{$1^{\text {re }}$ partie. - Etalonnage et dépouillement}

1. Le spectromètre. - L'appareil utilisé est un spectromètre à grilles fabriqué sous licence ONERA par la Société Générale d'Optique [1, 2, 3]. Ses principales caractéristiques étaient les suivantes lors des expériences :

- focale du collimateur $=2 \mathrm{~m}$,

- deux grilles de $28 \times 28 \mathrm{~mm}$, à dessins hyperboliques, de pas le plus fin $0,2 \mathrm{~mm}$, sur support de fluorine,

- réseau de diffraction "Bausch and Lomb» de $205 \times 128 \mathrm{~mm}$, à 73,25 traits $/ \mathrm{mm}$, utilisé dans le $3^{\mathrm{e}}$ ordre,

(*) Cet article fait partie d'une thèse de doctorat-d'ingénieur soutenue le 13 janvier 1971 à la Faculté des Sciences d'Orsay.
- globar de $300 \mathrm{~W}$ chauffé à $1500^{\circ} \mathrm{K}$,

- récepteur pneumatique ONERA, de surface sensible $14 \mathrm{~mm}^{2}$ et de NEP $1,7 \times 10^{-10} \mathrm{~W} / \sqrt{\mathrm{Hz}}$ à $10 \mathrm{~Hz}$,

- modulation sélective assurée par vibration à $5 \mathrm{~Hz} \mathrm{du}$ miroir parabolique.

Ainsi équipé, l'appareil travaille entre 1100 et $1500 \mathrm{~cm}^{-1}$ avec une résolution de l'ordre de $0,05 \mathrm{~cm}^{-1}$. Le gaz étudié est envoyé sous une pression de l'ordre de $1 / 10 \mathrm{~mm} \mathrm{Hg}$ dans la cuve vidée d'air ; l'épaisseur de gaz traversée est de $10 \mathrm{~m}$. Le fait de plonger le réseau dans le gaz à étudier ne provoque pas de décalage spectral détectable.

2. Conditions à remplir par l'étalonnage. - L'étalonnage en nombres d'onde sur un spectromètre à grilles pose les mêmes problèmes que sur un spectromètre à fente à haute résolution. Le point fondamental est d'enregistrer simultanément spectre à étudier et repères d'étalonnage, de façon à s'affranchir des perturbations aléatoires dues aux vibrations et aux flexions, dont l'effet est d'autant plus sensible que la résolution est plus élevée.

La solution la plus rationnelle consiste à enregistrer un spectre d'absorption suffisamment bien connu et situé dans le même domaine spectral que la bande étudiée [4]. De tels spectres n'existent pas encore autour de $1300 \mathrm{~cm}^{-1}$; il est donc nécessaire d'utiliser des spectres se rapportant à une autre région spectrale. Si les points de repères ainsi fournis sont trop distants les uns des autres, il faut adjoindre au spectromètre un système d'interpolation lié aux mouvements de l'appareil.

3. Enregistrement de points de repères de nombre d'onde. - La méthode utilisée, spécifiquement adap- 
tée au spectromètre à grilles, est basée sur le même principe que la méthode mise au point par P. Vermande [5]. On utilise les raies d'émission de diverses lampes spectrales entre 0,5 et $0,7 \mu$, raies dont les longueurs d'onde sont bien connues (voir par exemple les collections de "Proceedings of the I. A. U. »).

Le schéma de principe est celui de la figure 1. La grille d’entrée est attaquée en réflexion par le faisceau infrarouge, en transmission par le faisceau d'étalonnage. Entre les deux grilles, le chemin suivi par les deux faisceaux est identique. Le faisceau infrarouge est transmis par la grille de sortie vers le récepteur pneumatique, et le faisceau d'étalonnage est réfléchi vers un photomultiplicateur de type RCA 1P21. Le signal détecté par ce dernier est réinjecté sur la chaîne infrarouge juste avant la démodulation.

Lampe spectrale et photomultiplicateur sont disposés à l'extérieur de l'appareil. Pour le faisceau d'éta-

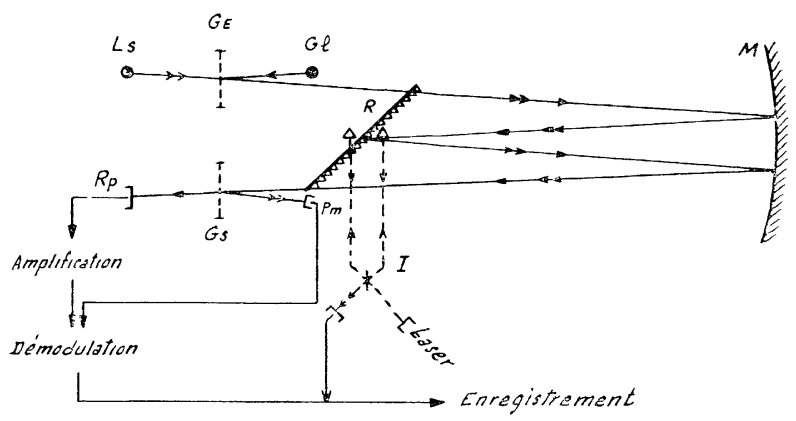

FIG. 1. - Schéma de principe du spectromètre avec le système d'étalonnage.

$\mathrm{M}=$ miroir parabolique vi- $\mathrm{R}=$ réseau de diffraction brant

$\mathrm{G}_{\mathrm{e}}=$ grille d'entrée

$\mathrm{G}_{\mathrm{s}}=$ grille de sortie

$\mathrm{G}_{1}=$ globar

$\mathbf{L}_{\mathrm{s}}=$ lampe spectrale

$\mathrm{R}_{\mathrm{p}}=$ récepteur pneumatique $\mathrm{P}_{\mathrm{m}}=$ photomultiplicateur

$\mathrm{I}=$ interféromètre de contrôle de la rotation du réseau



Pнoto 1. - Vue d'ensemble des optiques d'entrée et de sortie.

- Au fond, la cuve contenant la source infrarouge.

- En haut, la lampe spectrale étalon et les premiers miroirs du faisceau d'étalonnage.

- Au centre, le boîtier contenant le récepteur pneumatique (récepteur infrarouge).

- En haut à droite, le boîtier contenant le récepteur du faisceau d'étalonnage (photomultiplicateur). lonnage, le réseau travaille dans un ordre élevé (de 30 à 40).

Un tel système est théoriquement parfait à condition que le réseau soit dans le vide et que les deux faisceaux soient rigoureusement confondus entre les deux grilles. En fait une pression de quelques centimètres de mercure n'apporte pas de décalage entre les deux faisceaux. D'autre part le spectromètre à grilles ONERA, diaphragmé en son centre de symétrie par la monture du réseau, ne présente qu'une très légère distorsion et il a été vérifié que même un important décalage entre le faisceau infra-rouge et le faisceau d'étalonnage ne provoque pas d'erreur sensible.

4. Interpolation entre les raies d'étalonnage. Une solution classique consiste à monter sur le spectromètre un interféromètre dont la différence de marche rend compte du mouvement de l'appareil, y compris les micro-fluctuations; le montage dépend de la disposition du spectromètre $[4,6,7]$.

Le spectromètre à grilles ONERA est suffisamment court ( $2 \mathrm{~m}$ entre les grilles et le miroir) pour que l'influence des flexions d'ensemble soit négligeable. Par contre les mouvements du réseau et de l'enregistreur sont sujets à des irrégularités plus ou moins aléatoires, tout à fait détectables en comparant deux enregistrements du même spectre.

Le dispositif adopté est un interféromètre de Michelson dont les miroirs sont remplacés par des catadioptres trirectangles en verre solidaires de la plateforme du réseau (Fig. 2). La séparatrice est constituée par deux prismes de verre $30 \times 60^{\circ}$ collés par la grande face de l'angle droit, cette face ayant au préalable reçu un traitement semi-réfléchissant. La lumière est fournie par un laser He-Ne de faible puissance. L'axe de rotation du réseau, perpendiculaire au plan formé par les deux rayons interférents, est médiatrice du segment joignant les sommets des trièdres, ce qui permet aux deux rayons interférents de se superposer exactement quel que soit l'angle dont ont tourné les trièdres.

Les trièdres sont collés par leur grande face sur une lame de verre, et l’interféromètre esî réglé au voisinage

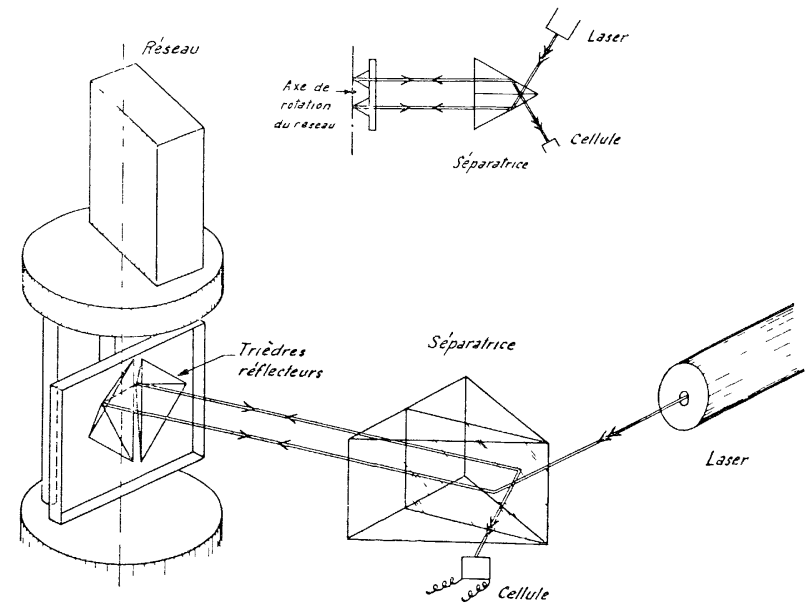

FIG. 2. 




Рното 2. - Interféromètre de contrôle de rotation du réseau.

- En haut, le réseau de diffraction. teurs.

- Au centre, au fond, sous le réseau, les deux trièdres réflec-

- Au premier plan, un peu sur la droite, la séparatrice avec ses boutons de réglage.

- En bas, un cercle divisé permettant d'apprécier grossièrement la position du réseau.

de l'ordre zéro ; ce montage très simple permet de faire travailler les trièdres dans les meilleures conditions.

La loi de répartition des franges en fonction du nombre d'onde peut être déterminée expérimentalement au moyen d'un développement limité. Le calcul montre toutefois que si l'interféromètre travaille au voisinage de l'ordre zéro et si les raies d'étalonnage sont à une distance angulaire inférieure à un demi-degré de rotation du réseau, on peut considérer que le nombre de franges est proportionnel à l'angle de rotation du réseau, l'erreur systématique étant inférieure à $0,001 \mathrm{~cm}^{-1}$ pour une course du réseau de $4^{\circ}\left({ }^{*}\right)$ de part et d'autre de la différence de marche nulle.

5. Détermination du nombre d'onde d'une raie spectrale. - Un spectre de travail se présente comme celui de la figure 3 :

-2 raies d'étalonnage de nombres d'onde $\sigma_{1}$ et $\sigma_{2}$ enregistrées dans les ordres $K_{1}$ et $K_{2}$;

- $n$ raies à mesurer le nombre d'onde $\sigma_{n}$ enregistrée dans l'ordre $K_{n}$;

- un réseau de franges d'interpolation comportant $m_{12}$ franges entre $\sigma_{1}$ et $\sigma_{2}$ et $m_{1 n}$ franges entre $\sigma_{1}$ et $\sigma_{n}$.

La relation de base est du type $A \sin \theta=K / \sigma, A$ étant un coefficient caractéristique de l'appareil et $\theta$ l'angle dont est incliné le réseau.

Les valeurs $\left(\sigma_{1}, K_{1}\right)$ et $\left(\sigma_{2}, K_{2}\right)$ permettent de déterminer les angles $\theta_{1}$ et $\theta_{2}$. On calcule alors la valeur de $\theta_{n}$ la relation :

$$
\theta_{1}-\theta_{n}=\frac{m_{1 n}}{m_{12}}\left(\theta_{1}-\theta_{2}\right)
$$

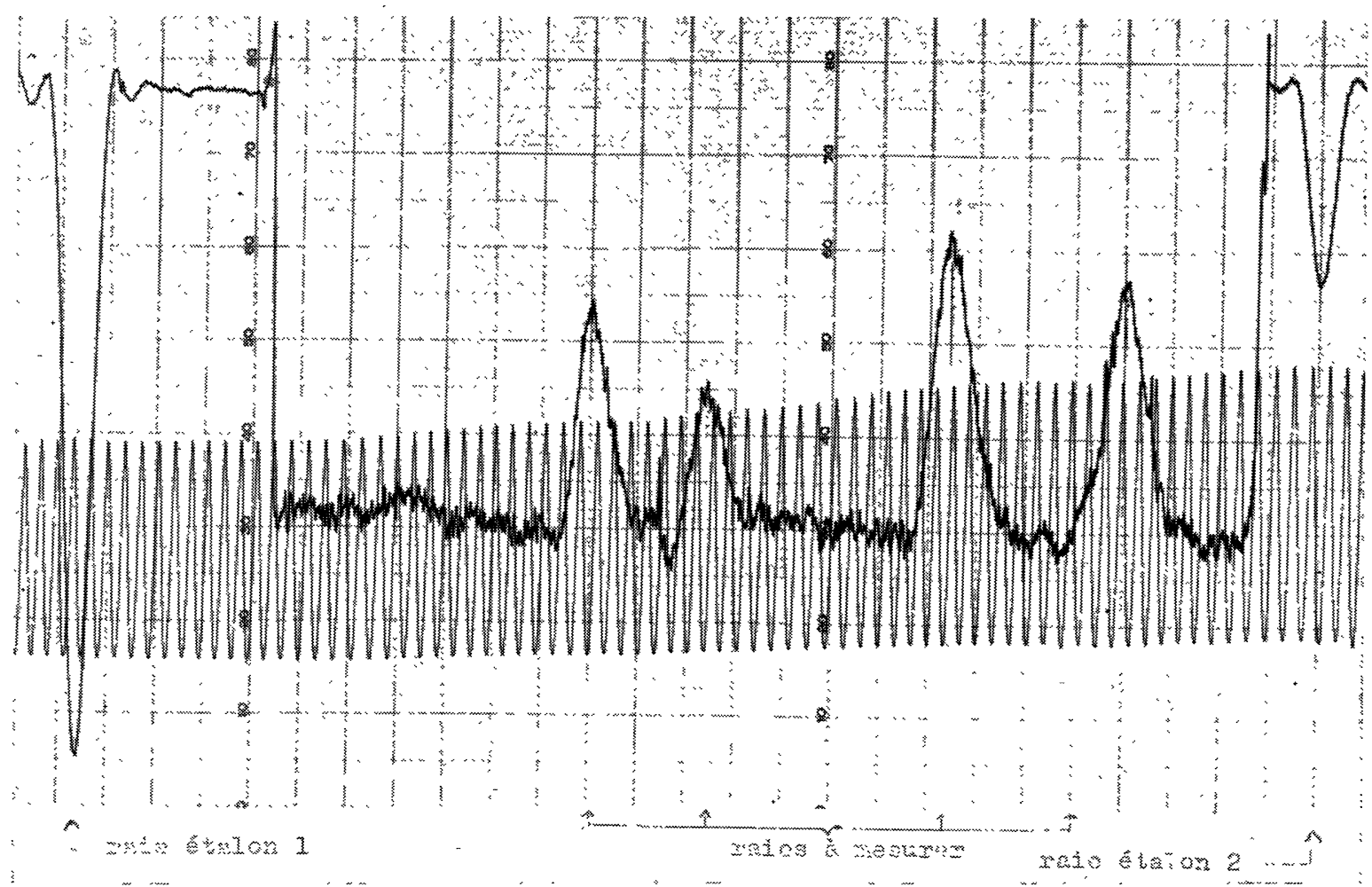

FIg. 3. - Exemple de spectre de travail avec deux raies d'étalonnage.

$\left(^{*}\right)$ Il convient de remarquer qu'en faisant travailler l'interféromètre dans un ordre convenable, on pourrait avoir une loi de répartition des franges d'interpolation rigoureusement linéaire en longueur d'onde. Mais pour les angles d'inclinaison du réseau très élevés, le montage mécanique devrait être plus complexe afin de faire travailler les trièdres à peu près normalement en faisceau laser. 
On déduit enfin du couple $\left(\theta_{n}, K_{n}\right)$ la valeur de $\sigma_{n}$. Ce calcul se programme très simplement sur un ordinateur.

Un contrôle de la méthode d'étalonnage a été effectué à l'aide du spectre d'absorption de la molécule $\mathrm{CO}$ vers $4000 \mathrm{~cm}^{-1}$, dont les valeurs des nombres d'onde sont bien connues [8].

Dans le domaine spectral $1200-1400 \mathrm{~cm}^{-1}$, la précision atteinte est de $0,004 \mathrm{~cm}^{-1}$ pour les raies intenses et isolées. $2^{\mathrm{e}}$ partie. - Présentation de quelques résultats relatifs à la bande fondamentale $v_{4}$ du méthane

Le spectre complet de la bande pourra être consulté par ailleurs [9]. Ci-dessous figurent quelques exemples caractéristiques, ainsi qu'une comparaison avec des résultats antérieurs $[10,11,12]$, tant théoriques qu'expérimentaux.

La figure 4 représente la tête de la branche $Q$, qui est la région la plus dense en raies de toute la bande ; les nombres d'onde associés sont les suivants $\left(\mathrm{en}^{-1}\right)$ :



Fig. 4. - Bande $v_{4}$ du méthane. Tête de la branche $Q$.

Dans cette bande, peu de valeurs ont été mesurées par Rao et Migeotte, en raison du manque de résolution des appareils utilisés.
La figure 5 représente le groupe de deux raies $\mathrm{R} 2$, difficilement séparées par le spectromètre; les nombres d'onde associés sont les suivants : 




FIG. 5. - Bande $v_{4}$ du $\mathrm{CH}_{4}-\mathrm{R} 2$.

ONERA

(mesuré)

1322,109

1322,162

$$
\begin{aligned}
& \text { Rao-Moret-Bailly } \\
& \text { (mesuré) } \quad \text { (calculé) }
\end{aligned}
$$

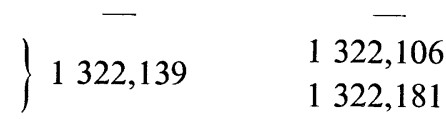

Kyle

(calculé)

1322,03
1322,13
Migeotte-Neven-Swensson (mesuré)

spectre laboratoire solaire

1322,15

Le groupe de trois raies $\mathrm{R} 3$ apparaît nettement dissocié sur la figure 6:

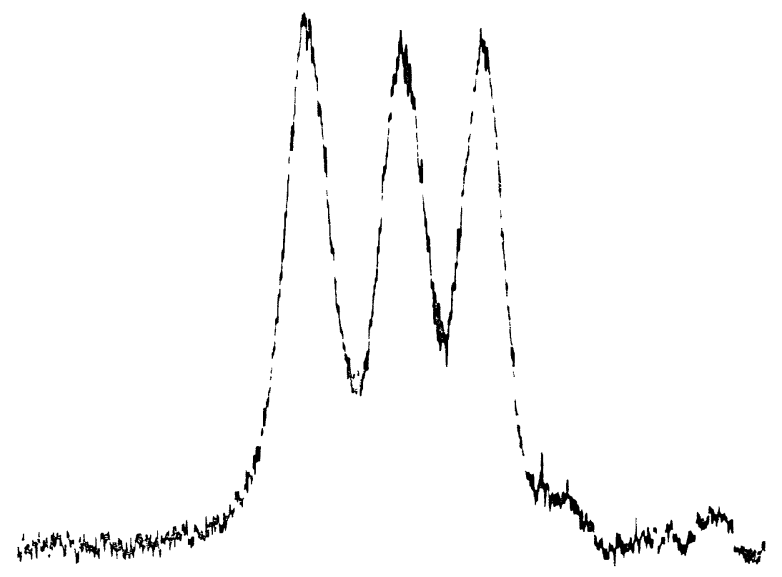

Fig. 6. - Bande $v_{4}$ du $\mathrm{CH}_{4}-\mathrm{R} 3$. 
Considérons enfin le groupe R7, composé de six raies (Fig. 7) :



Fig. 7. - Bande $v_{4}$ du $\mathrm{CH}_{4}-\mathrm{R} 7$.

O NERA

$\begin{array}{ll}1 & - \\ 346,328 \\ 1346,573 \\ 1346,735 \\ 1347,052 \\ 1347,918 \\ 1348,034\end{array}$

Rao-Moret-Bailly

1346,352
1346,648
1347,042
1347,946

1346,432

1346,582

1346,782

1347,009

1347,764

1347,952

Kyle
1344,28
1344,77
1345,09
1345,68
1347,28
1347,49

Migeotte-Neven-Swensson
$\begin{array}{rr}1346,31 & 1346,25 \\ 1346,61 & 1346,60 \\ 1346,98 & 1347,02 \\ 1348,00 & 1348,00\end{array}$

De ceci, il ressort que les valeurs mesurées à l'ONERA semblent mieux correspondre aux valeurs précédemment mesurées qu'aux valeurs calculées par les divers auteurs.

Un nouveau calcul des constantes moléculaires du méthane s'impose donc, d'autant plus que la branche $Q$ peut fournir toute une série de nouvelles équations.

Ce travail, effectué à partir des spectres réalisés à I'ONERA, est en cours au Laboratoire de Spectroscopie Moléculaire de la Faculté des Sciences de Paris.

Conclusion. - Le gain important de luminosité apporté par l'emploi de grilles dans un spectromètre infrarouge permet d'atteindre la résolution maximale des réseaux utilisés, avec une vitesse de balayage relativement grande. Par adjonction de dispositifs optiques d'étalonnage, la précision de la graduation de l'échelle spectrale des enregistrements est maintenant compatible avec les plus hautes résolutions permises.

C'est ainsi qu'ont été mesurés les nombres d'onde des raies de la bande fondamentale $v_{4}$ du méthane entre 1225 et $1490 \mathrm{~cm}^{-1}$. La précision propre à l'étalonnage est estimée à $0,003 \mathrm{~cm}^{-1}$. La qualité de ces résultats va permettre aux théoriciens de la spectroscopie moléculaire de reprendre les calculs des constantes de la molécule de méthane et d'en établir un nouveau modèle conforme à l'expérience.

D'autres expériences sont en cours avec le spectromètre à grilles dans l'infrarouge plus lointain au voisinage de $670 \mathrm{~cm}^{-1}$. Dans ce domaine, le récepteur pneumatique est remplacé par une cellule en germanium dopé au cuivre refroidie par hélium liquide, dont la détectivité est meilleure.

D'autre part une série d'essais destinés à perfectionner le processus de l'acquisition et du traitement des données fournies par l'appareil est envisagée. Un système d'enregistrement sur bande perforée est en cours. de mise au point. Le traitement des informations pourra ainsi être directement assuré par un ordinateur.

Bibliographie

[1] Girard (A.), Appl. Opt., 1963, 2, 79.

[2] Girard (A.), “Etude d'un Spectromètre à Modulation Sélective ), Publ. no 117, 1967, ONERA.

[3] Notice technico-commerciale sur le Spectromètre à Grilles licence ONERA-JOBIN \& YVON SGO, 26, rue Berthollet, 94, Arcueil.

[4] Moret-Bailly (J.), C. R. Acad. Sci., Paris, 1969, 268 B.

[5] Vermande (P.), J. Physique, 1968, 29, 1041.

[6] Henry (L.) et Valentin (A.), communication privée.

[7] COLE (W. L.), "A Michelson interferometer for Detecting grating Table Rotation ), NASA, CR $101450,1969$.
[8] Narahari Rao (K.), Humphreys (C. J.), Rank (DH.), ( Wavelength Standards in the Infrared ), Academic Press, New York, London.

[9] Botineau (J.), à paraître.

[10] Moret-Bailly (J.), Thèse Ed. Rev. Opt., 1961, A 3762.

[11] KYLE (T. G.), ( Line Parameters of the Infrared Methane Bands "), U S A F Res. Lab. AFCRL, 1968, 68-0521.

[12] Migeotte (M.), Neven (L.), Swensson (J.), “The Solar Spectrum from 2,8 to 23,7 microns ), Part. II, Mem. Soc. Roy. Sc. de Liège, 1957, 2. 